\title{
Theories on the Formation and Evolution of Spiral Galaxies \& Rate of Universal Expansion
}

\author{
Author contact: M2Materials@,Outlook.com
}

\begin{abstract}
:
Spiral galaxies like the Milky Way present fascinating behavior owing to the beautiful symmetry and well-defined organization of their spiral arms and typical 30:1 ratio of their width to depth. Such symmetry is another celestial example that appears to violate the law of entropy favoring uniform spatial dispersion of matter and energy with time, in which case a random distribution of stars and solar systems throughout the vast 4-D space-time continuum would have been the expected result over the billions of years since galactic birth. The plausible scientific explanations are of course forces that worked to achieve such spiral symmetry and unusual $\sim 2 \mathrm{D}$ planar kinematics. While convention attributes such kinematics to gravitational collapse along the axis of accretion rotation, this paper proposes that the forces at work are the kinetic forces of immense accretion-matter vortex at the galactic core combined with colossal electromagnetic force-fields spawned within (previously described and classified as the NOEMKV force-field) during the formation period, and extends this analysis to the formation of geometric configurations of galaxies with a focus on the spiral arms of the Milky Way as the primary model. An interesting postulate on the rate of expansion of the Universe is also discussed, derived directly from the galactic NOEMKV formation and evolution forces.
\end{abstract}

Keywords: spiral galaxies, elliptical galaxies, galaxy formation, galaxy evolution, celestial kinematics, magnetic compression, electromagnetic fields, fire-tornadoes, vortex forces, dark matter, dark energy, expansion of the universe

\section{Background and the Physics of Model Development:}

Spiral galaxies occur so frequently that "the Universe seems to delight in building them." In two previous publications ${ }^{2,3}$ the NOEMKV (Net Omnipresent ElectroMagnetic Kinetic-Vortex) force-field theory was proposed as the primary forces involved in the formation and evolution of stars, black holes, and galaxies and in particular as an explanation for the unusual $\sim 2 \mathrm{D}$ planar kinematics of solar systems and galaxies. As another example of the violation of the law of entropy, the symmetry of spiral arms in galaxies is an excellent area of focus to apply the NOEMKV force-field physics to highlight concepts and details of the theory while hopefully contributing to the understanding of celestial kinematics and galactic evolution.

In the preceding paper "Theories on the Formation and Evolution of Black Holes \& Galaxies" it was hypothesized the majority of the matter in the Milky Way was part of the galactic accretion vortex during the galactic formation period and gradually spread out over the billions of years since formation. This would fulfill the criteria for the net force-field vector of proposed tremendous magnetic pressure and kinetic vortex forces of the galactic core's NOEMKV forcefield to keep the majority of the galactic matter constrained to $\sim 2 \mathrm{D}$ planar orbital kinematics, even though today the galactic magnetic field is only $1 * 10^{-6}$ that of the Earth's magnetic field as measured on the surface of the Earth ${ }^{4}$. The apparent weakness of the galactic magnetic field is the main reason it is not given much credit in the formation of galaxies and stars, and mass-based 
gravity-effect theories are favored instead. The previous publications and continuing with this paper propose that the strength of the electromagnetic field and associated magnetic pressure have been significantly under-estimated in magnitude and effect during the formation period of stars and galaxies billions of years ago.

Assuming the background galactic magnetic field is being measured correctly at present on Earth, the proposed kinetic and electromagnetic forces of the galactic NOEMKV field could have been performed on the bulk of the Milky Way's matter (the portion that exhibits 2D planar orbital kinematics about the galactic core) under any or all of the following conditions:

(1) The bulk of the galactic matter was closer to the galactic core during the formation process and part of the galactic-core accretion vortex during that time, and subject to the galactic NOEMKV force-field at that time; the matter expanded over billions of years to the present-day Milky Way as the galactic NOEMKV force-field declined in magnitude

(2) The strength of the magnetic field (and associated magnetic pressure) of the galactic NOEMKV force-field acted over a much wider distance during the formation period than it does today

(3) The chain-link induction of any electromagnetic field ${ }^{5}$ and in this case the galactic core's electromagnetic force-field combined with conservation of angular momentum of the accretion-matter vortex could have imparted the net-force vector NOEMKV kinematics of the galactic core to matter that spiraled out of the galactic core (this matter retained the general kinematics of the galactic core as it spread out over the $\sim 2 \mathrm{D}$ galactic plane)

This paper will focus primarily on conditions (1) and (3).

In accordance, the proposed NOEMKV force-field and its effect on the formation and evolution of spiral galaxies will be demonstrated in two primary kinematic models as follows:

Kinematic Model 1: assumes the bulk of the matter in the galaxy (that formed orbits in the $\sim 2 \mathrm{D}$ galactic plane) was part of the galactic core vortex during the formation period. The vortex is modeled geometrically as accretion matter forming a cylindrical shell rotating around a vortex eye, and was formed based on heat-driven forces (akin to a fire-tornado on Earth) and electrostatic attractions from the friction of gas and dust collisions. At the weakest point of the NOEMKV force-field (furthest distance from the core Z-central point of greatest vortex intensity and at the surface boundary conditions with cold space) accretion matter systematically escaped primarily as gas/dust/plasma. Spiral arms formed from a critical point of weakest force at the top and bottom of the cylindrical vortex faces of the Milky Way's galactic core. This is the proposed to be the primary route of escaping matter that formed the arms and eventually the billions of stars of the Milky Way.

The shape of the spiral arms is proposed to be symmetric about the galactic core's cylindrical symmetry for this model, with spiral arms that were very closely packed during the formation period and gradually expanded radially outward over billions of years thereafter. This radial expansion is represented as occurring from decay in the galactic core's NOEMKV force-field in this model. As more and more matter escaped the galactic core and available energy to drive the fire-tornado heat and electrostatic-friction vortex engine decreased, both the kinetic and 
electromagnetic force-field components of the galactic core NOEMKV decreased. With less matter, less collisions, and lower net vortex velocity the electric field would decrease and so to would the magnetic field (and associated magnetic pressure) it spawned. This would lead to decreased influence of NOEMKV on the spiral arms, leading to expansion of the arms away from the core.

As in the star-formation and galactic-formation modeling in the previous publications, despite this decay the work of the galactic core NOEMKV force-field was already done in the formation period in shaping the kinematics of the spiral arms. The Milky Way fire-tornado vortex still rages as fuel to drive the heat and electrostatic vortex is still available though at a much lower extent than billions of years ago during galactic formation, so in this model the galactic core is a cylindrical shell vortex rotating around an eye (the black hole Sagittarius A* at the center of the Milky Way), wherein the black hole is largely void of matter and mainly serves as an important inner surface for the electromagnetic field components of NOEMKV to form, traverse, and rotate and is not a singularity of enormous mass-density of conventional theory. In the previous galaxyfocused paper ${ }^{6}$, the cylindrical galactic vortex was modeled as the approximate size of the mostintense region of the galactic core in the present era (diameter of 3,000 light-years (LY) and height of 4,000 LY); here an additional 1,000 LY is added to the diameter as an approximation for the additional mass that is modeled as part of the galactic vortex during formation (that later spread out to be the spiral arms). Kinematic Model 1 then becomes:

\section{Figure 1: Kinematic Model 1 of the Milky Way During the Spiral-Arm Formation Period}

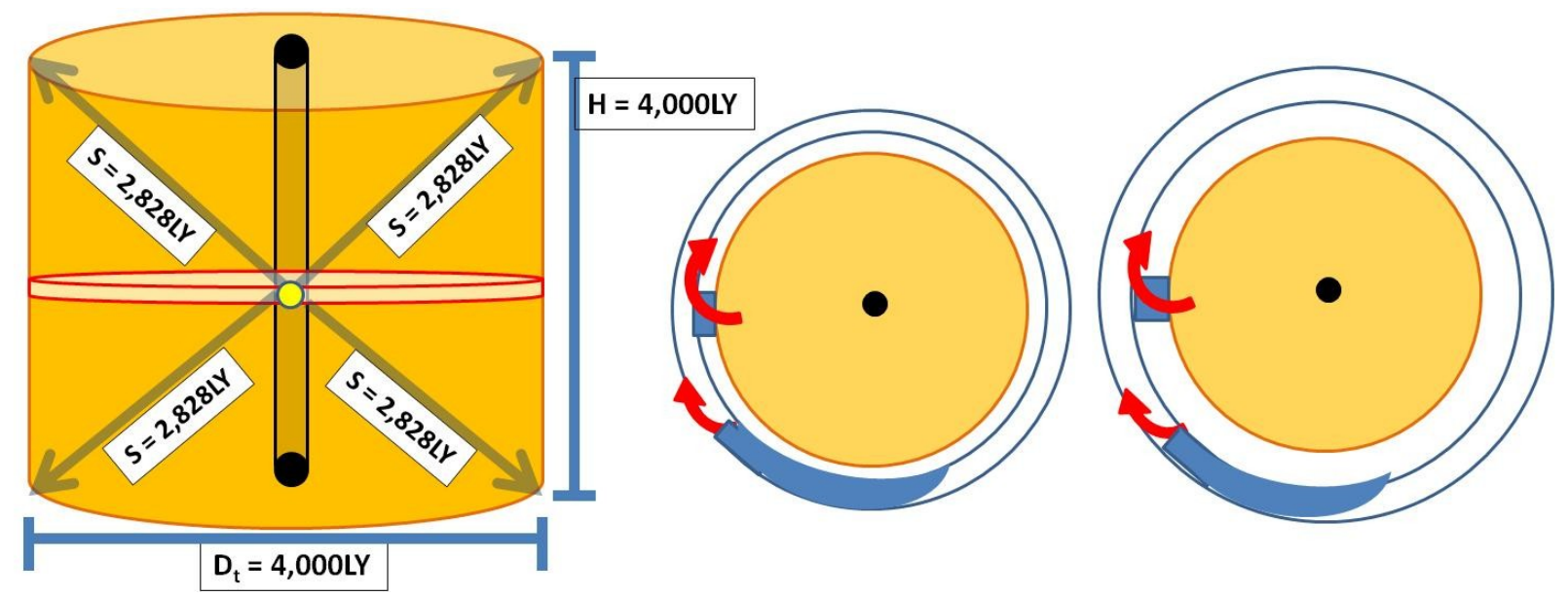

In the depiction on the left (tilted side view), the line segments between the Z-central point (marked with a yellow circle in the Z-central $\theta$-plane) to any point on the outside perimeter of the cylinder's top and bottom faces marks the distance furthest away from the Z-central point (the hypotenuse of a right triangle with height of 2,000 light-years (LY) and leg of 2,000 LY equals Side $\mathrm{S}=2,828 \mathrm{LY}$ ). The depiction on the right (top or bottom view looking straight up or straight down the $\mathrm{Z}$ axis) shows a spiral arm (blue lines) of systematically escaping galactic accretion matter originating from a point on the outside perimeter of the top (or bottom) faces of the cylinder with spiral cylindrical symmetry retained though with gradual radial expansion over time depicted by the expanding spiral arms. 
With such perfect symmetry, it would be difficult to justify the points along the top and bottom faces of the cylinder that would be the "critical point" of force weakness where the systematic escape of accretion matter commences to form the spiral arms. Plausible reasons are given below in Kinematic Model 2:

Kinematic Model 2: Same general concepts as Model 1 except here the galactic core is stretched at the top and bottom boundary surface regions from a cylinder to an ellipse, where the major-axis of the ellipse becomes the point at which NOEMKV is furthest from the core (and weakest) and therefore the critical point at which accretion matter systematically escapes to form the spiral arms. The construct below assumes a stretch along the major axis resulting in a diameter of 22,500 light-years (corresponding to the Milky Way's elliptical Galactic Bar at the core of the galaxy) based on estimates in the NASA map of the Milky Way ${ }^{7}$ and a minor axis of 4,000 light-years (corresponding to Kinematic Model 1 for the additional mass). Height (depth) will assume to remain the same as Kinematic Model 1 (4,000 LY) matching the approximation for the depth of the galactic bulge at the core of the Milky Way. The diameter of the most intense central vortex core region (assumed to be the galactic bulge) retains cylindrical geometry of 4,000 LY diameter and 4,000 LY height of Kinematic Model 1.

Figure 2: Kinematic Model 2 of the Milky Way During the Spiral-Arm Formation Period

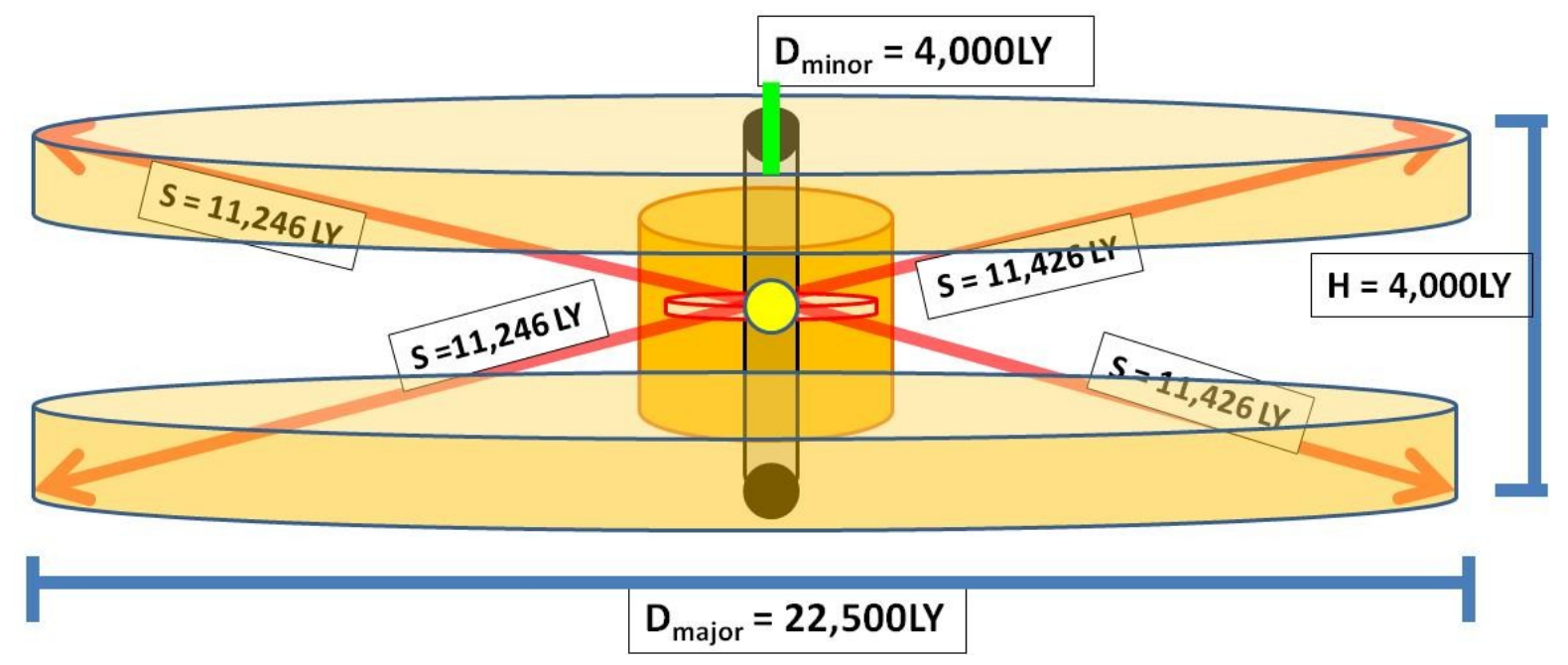

The hypotenuse from the Z-central point (yellow circle) to the furthest point on the major axis of the top and bottom elliptical faces is 11,426 LY. Comparing this to the hypotenuse from the Zcentral point to the closest point on the minor axis of the top and bottom elliptical faces (hypotenuse $=2,828 \mathrm{LY}$ which is a factor of $\sim 4$ times closer) it becomes clear that the NOEMKV force-field would be much weaker along the major axis. This is why the model proposes that the systematic escape of galactic accretion matter that formed the spiral arms originated from the galactic core vortex along the major axis of elliptical top (or bottom) boundary regions during the spiral-arm formation process of the Milky Way (and any similar spiral galaxies). 
The shape of the spiral arms and distance from the arms to the galactic core are proposed to have occurred based on force-balances in different Zones depicted and discussed below:

Figure 3: Zones of Force-Balance in Shaping the Spiral Arms for Kinematic Model 2

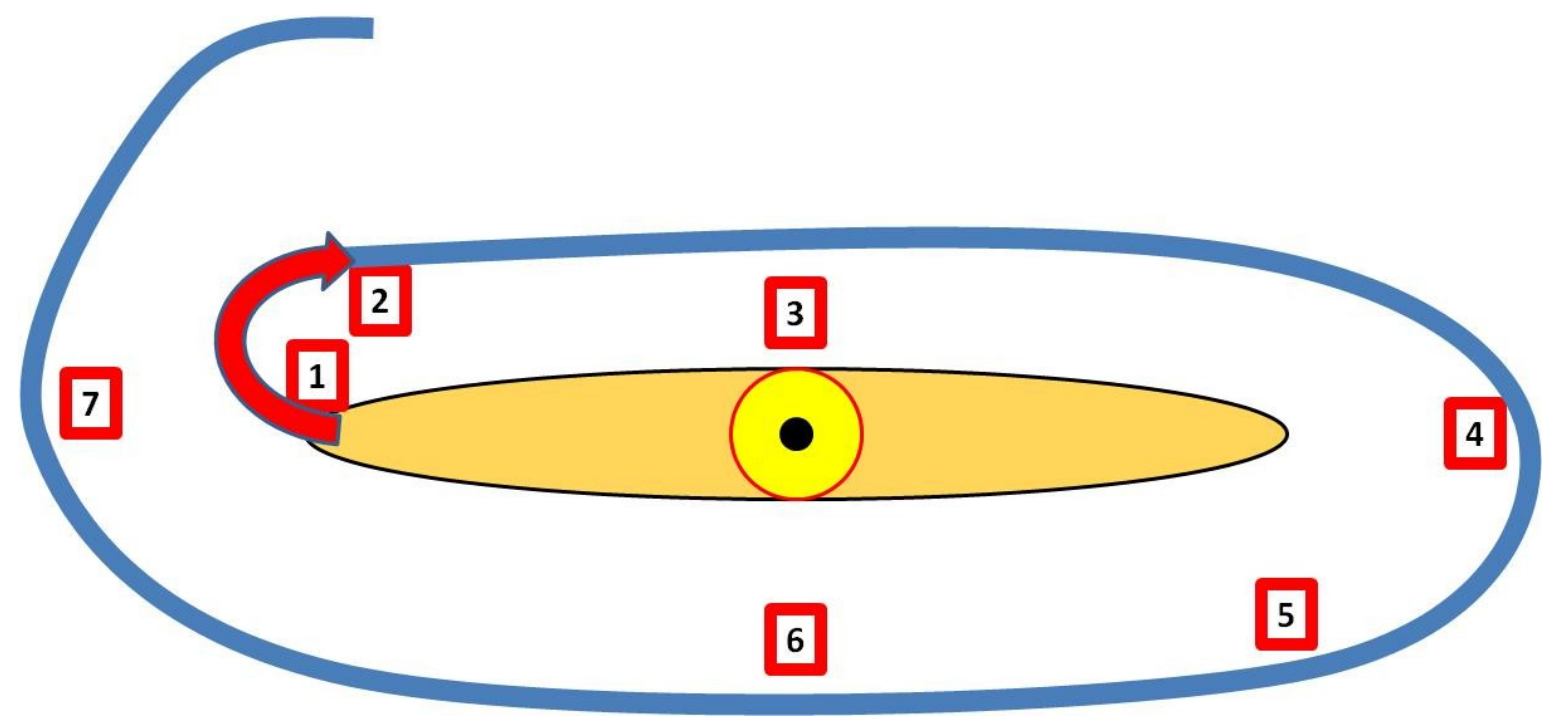

The view of Figure 3 is from either the top or the bottom elliptical faces of the Milky Way in Kinematic Model 2 (looking straight down in $-\hat{Z}$ or straight up in the $+\hat{Z}$ axis). The numbers in the red boxes are the Zones of force balances that are proposed to dictate the general shape of spiral arm (blue line) and its distance from the galactic accretion cylinder vortex with stretched elliptical top and bottom boundary faces (pale orange with black border to cold space) and mostintense galactic vortex core (yellow cylinder with black hole indicating the central vortex eye). The proposed physics and force-balance zones are:

Zone 1: The systematic escape of galactic accretion matter begins at the major axis (on either the top or bottom elliptical faces) where the NOEMKV force-field vector is furthest from the ZCentral point of the galactic core vortex. During this galactic formation period, the escaping matter remains discrete particles of highly conductive and magnetic gas/dust/plasma that will begin to generate its own electromagnetic field as it moves through space in addition to the chain-link tie to the electromagnetic field of the galactic core from whence it came (and is still attached in terms of continuously escaping accretion matter forming relatively continuous spirals of matter). The escape begins (and continues) whenever the centrifugal force of the matter in the $\theta$-plane is greater than the net NOEMKV force-field component in the $\theta$-plane at the location of the escape. The NOEMKV force-field components in the $\theta$-plane are:

(1) the centripetal force of the vortex

(2) the chain-link electromagnetic field induction between the escaping matter and the galactic core matter from which the escaping matter becomes an extension as spiral arm

(3) the electromagnetic (EM) field/wave interaction between the EM field/wave of the escaping matter and the EM field/wave of the galactic core across the space between them (the "Omnipresent" in NOEMKV refers to the continuous ElectroMagnetic and Kinetic-Vortex force-fields within the galactic core vortex accretion matter and the continuous 4-D space-time propagation of that force-field as a wave that traverses 
through empty space as an EM wave at light speed with magnitude and direction determined by the net vector of the galactic core's NOEMKV force-field, and the harmonic interactions of these waves as constructive, destructive, or neutral depending on location).

In Zone 1 the centripetal force is clearly at a minima due to furthest distance from the Z-Central point. The $\theta$-plane EM force-field is also predicted to be at a minima at this location for the same reason; the two minima create the critical weak point from which galactic core accretion matter systematically escapes. The proposed magnetic pressure of the galactic NOEMKV force-field (acting primarily in the $+\hat{Z}$ and $-\hat{Z}$ axis and forming continuous looping lobes all around the galactic accretion $)^{8}$ is however enough to keep the systematically escaping matter constrained within the galactic plane when it exits the core; combined with the direction and speed (velocity) of the escaped matter, the kinematics of escaping matter are established as $\sim 2 \mathrm{D}$ planar and with velocity determined (from conservation of angular momentum at the moment in time the escaping matter leaves the galactic core) even as it spreads throughout the galactic plane.

Zone 2: The direction of the systematically escaping matter of Zone 1 is such that the matter still retains the elliptical curvature of motion about the $\theta$-plane from which it escaped; it is a "narrow escape" (not a forced ejection) as centrifugal force just exceeds the $\theta$-plane NOEMKV forces to escape at the critical point and form a semi-bound parabolic orbit as the matter continues to be under the influence of the NOEMKV forces that organized and imparted kinetic/EM energy to it (when it was part of the galactic accretion vortex) and which it is still in proximity to (as it has "just escaped" in cosmic time during the formation process). This is observed in Zone 2, where the centripetal force component and EM interaction cause an inflection back toward the galactic core and prevent an unbound parabolic orbit from occurring. This inflection would have probably been magnified via the interaction of the EM field/wave of the escaping matter in Zone 2 and constructive interference with the EM field/wave of the galactic core as the spiral arm's orbital kinematics get closer to the galactic core's NOEMKV field; especially during the early formation period when it is predicted this NOEMKV was the strongest and the spiral arms of the Milky Way were still discrete matter (not yet stars) and more tightly bound to the galactic core.

Zone 3: Same forces as Zone 2 and noting that the NOEMKV force-field is at a local maxima at this boundary region of the elliptical galactic accretion faces because the distance to Z-central is a minima at the minor axis of the elliptical faces. The most powerful cylindrical galactic core vortex region (the global maxima of the galactic NOEMKV force-field) is in-between the top and bottom elliptical boundary faces at this location and imparts the local maxima to the top and bottom boundary faces due to proximity minima.

Zone 4: as the spiral arm matter approaches the next major axis, the galactic NOEMKV forcefield again approaches the local minima and the orbit of the escaping matter becomes stretched, mimicking the stretch of the galactic core's elliptical faces.

Zone 5: same factors as Zone 2. Same directional components though reduced magnitude as distance of the escaping matter (spiral arm) from the core increases, the contraction-inflection forces decrease. The reduction in contraction-force will make the arms more circular (lower eccentricity) as distance from the galactic core increases. 
Zone 6: same factors as Zone 3, though at reduced magnitude as distance is now further between the escaping matter (spiral arm) and the galactic core's minor axis.

Zone 7: same factors as Zone 4, and the cycles 2-7 repeat thereafter further out in the galactic plane

For both Kinematic Models, computational models for an expanding spiral are being derived to fit the kinematic constraints of the spiral arms of the Milky Way of present-day observations and contracted back in time to where it is assumed all of the present-day $\sim 2 \mathrm{D}$ galactic planar matter was part of the galactic core accretion vortex. The computational models will be the mathematical expression of dynamic 4-D space-time behavior of:

- The magnitude of the galactic core's NOEMKV force-field at the time of galactic formation (when the portion of matter that exhibits $\sim 2 \mathrm{D}$ planar orbital kinematics about the galactic plane is assumed to be part of the galactic core vortex)

- The global minima of the NOEMKV force-field (at the furthest distance from the ZCentral point because the magnetic field and kinetic field/force of the vortex both decreasing as $1 / \mathrm{r}$ from the core of the vortex); this is selected as the location where the spiral arms originate

- The centrifugal force needed to just overcome the attraction of matter to the galactic core vortex (centripetal force and electromagnetic attraction in the $\theta$-plane at the location where matter escapes to form the spiral arms).

- The contraction-inflection of escaping matter back toward the galactic core as the orbiting spiral matter approaches a local maxima in NOEMKV force-field strength at the minor axis of the boundary-face ellipse

- The effect of decreasing galactic / spiral arm NOEMKV force-field interaction as the distance to the spiral arms increases

- The effect of the balance of centrifugal force and conservation of angular momentum of the kinetic components of NOEMKV imparted to the escaping matter and net constructive/destructive interference of the EM components (of NOEMKV field of the galactic core and NOEMKV field of the spiral arms) at any instantaneous moment in time (differential basis) and the net (integral basis) which results in the semi-bound shape of the spiral arms and the determination of the kinematics (orbital velocity, trajectory, and $\sim 2$ planar characteristic) of escaping accretion matter at the time it escaped the galactic core and evolution thereafter

- The decay of the NOEMKV force-field as matter systematically escaped over time leading to expansion of the spiral arms from the galactic core over time

One caveat to the modeling is that the escaping matter eventually formed billions of stars that create their own localized kinematics to a certain extent, in accordance with NOEMKV forcefield theory applied to the localized fire-tornado vortex of accretion matter that formed stars ${ }^{9}$. Remarkably the billions of stars in the Milky Way still retain kinematics in the $\sim 2 \mathrm{D}$ galactic plane, which suggests that all such matter was strongly influenced by the galactic core NOEMKV force-field if NOEMKV force-field theory is the primary mechanism of galaxy and star formation. 
A well-known paradox of traditional gravity-theory offers additional support to the postulate all of the $\sim 2 \mathrm{D}$ planar galactic matter was greatly influenced by the galactic-formation period NOEMKV force-field; the stars in the outer regions of spiral galaxies have been observed to orbit at the same speed as stars closer to the center (and much faster than would be predicted based on Newton's Laws of gravity for the distance they are from the galactic core), as measured and reported originally by the outstanding work of Vera Rubin and many other astronomers since her pioneering work ${ }^{10}$. Such anomalies are utilized as the primary argument for the existence of "dark matter" providing additional mass-based gravitational force in the outer regions of galaxies. As an alternative to "dark matter" and mass-based gravitational force-theory in general, NOEMKV force-field theory predicts three complementary facets to explain this anomaly:

(1) If the bulk of galactic matter was closer to the galactic core during the formation process and part of / greatly affected by the galactic core fire-tornado vortex of accretion matter as suggested, the imparted angular momentum at the time it escaped the galactic core vortex was much greater and can result in a faster orbit (compared to mass-gravity force theory) due to conservation of that angular momentum, even as that matter spread out from the core region in the $\sim 2 \mathrm{D}$ galactic plane.

(2) The chain-link induction nature of electromagnetic fields enables a particle-to-particle connection between the net NOEMKV force-field of the galactic core to have extended into the escaping matter spiraling out of and about the core; this connection is predicted to have been the strongest during the formation period and would have helped impart the strong vortex kinetic force (that will have remained conserved) and semi-bound expanding orbital trajectory to the spiral-arm matter. During the formation period and a substantial period of cosmic time thereafter, the escaping matter would have likely remained highly electrically and magnetically conductive discrete gas/dust/plasma accretion matter so that the net electromagnetic and kinetic forces of the galactic core NOEMKV field will have easily been imparted

(3) The localized NOEMKV force-field of star formation can impart faster velocity to the star accretion matter according to the strength of the fire-tornado vortex of accretion matter that forms each star. The strength and velocity of the fire-tornado vortex is a function of (A) that matter's initial angular momentum (proposed to have been imparted by the NOEMKV force-field of the galactic core it was originally part of as described above) plus (B) the additional velocity driven by available fuel for the heat-engine of the star's fire-tornado vortex. Localized regions of space with higher fuel availability (e.g. more available hydrogen) and electrostatics (e.g. more dust) can create stronger vortex/EM fields and impart greater velocity to the star's accretion matter (and to the star that the accretion matter forms upon coalescences, via conservation of the localized star NOEMKV force-field's angular momentum).

Established theory based on observation of the cosmos offers additional support to NOEMKV force-field theory. All matter in the universe is believed to have been accelerating rapidly away from each other (in the Big Bang) 13.7 billion years ago then slowed in expansion rate (decelerated) till about 5-6 billion years ago based on careful observations of distant supernova ${ }^{11,12}$. Assuming the Big-Bang theory is correct, then all universal matter would have had directional momentum imparted to initially expand radially away from each other unless/until something caused it to slow down. 
NOEMKV theory proposes that when collisions of matter generated sufficient heat and electrostatic attraction, the proposed fire-tornado vortex formations of galaxies began. The organization of matter into galaxies would create additional velocity/momentum vector components of the rotating vortex matter (instead of just "everything radially away from the BigBang") and slow the Big-Bang radial momentum vector during these galactic formation processes. The galactic formation processes is believed to have begun in the range of $\sim 100$ million years after the Big-Bang for the most ancient galaxies, and NOEMKV theory predicts the majority of galaxies formed from then through 5-6 billion years ago and reduced the Big-Bang radial momentum vector accordingly throughout this formation period. Around 5-6 billion years ago, the integral sum of all galactic NOEMKV force-fields began their decay (enabling things like spiral arms and hundreds of billions of stars in the Milky Way and similar galaxies expanding in space, as detailed above and which cosmic time-frame is well supported by the formation of our solar system's Sun $\sim 4.5$ billion years ago); instead of a reduction in the BigBang radial momentum vector that galaxy-formation had been causing, the expansion of galaxies as their NOEMKV force-fields decreased and evolved now adds to the Big-Bang radial momentum vector. Throughout this process the Big-Bang radial momentum vector remained (and remains) the dominant momentum force such that all matter (even that organized in galaxies) moved apart at all times, with the current rate of expansion (the Hubble Constant) somewhere in the range $72 \mathrm{~km} / \mathrm{s}$ per 3.26 million light years ${ }^{13}$.

Taking this a bit further, the same decay of net NOEMKV galactic force-field which led to the expansion of the Milky Way (from the bulk of matter being in an accretion vortex to the systematic escape of matter that formed spiral arms that continue to expand away from the galactic core) would occur in all regions of space where galaxies formed, for the exact same reasons. One portion of a distant galaxy's spiral arm could be expanding toward Earth (when it's expansion motion was aligned toward Earth) while the 180 degree about portion would be expanding away from Earth, The net "Universal Expansion Rate" would be a function of the net momentum vector (Universal vs. localized Galactic momentum) depending on position as depicted in Figure 4:

\section{Figure 4: Net Rate of "Universal Expansion Rate" Accounting for Galaxy Expansion Momentum vs. Universe Momentum Expansion for an Observer on Earth}

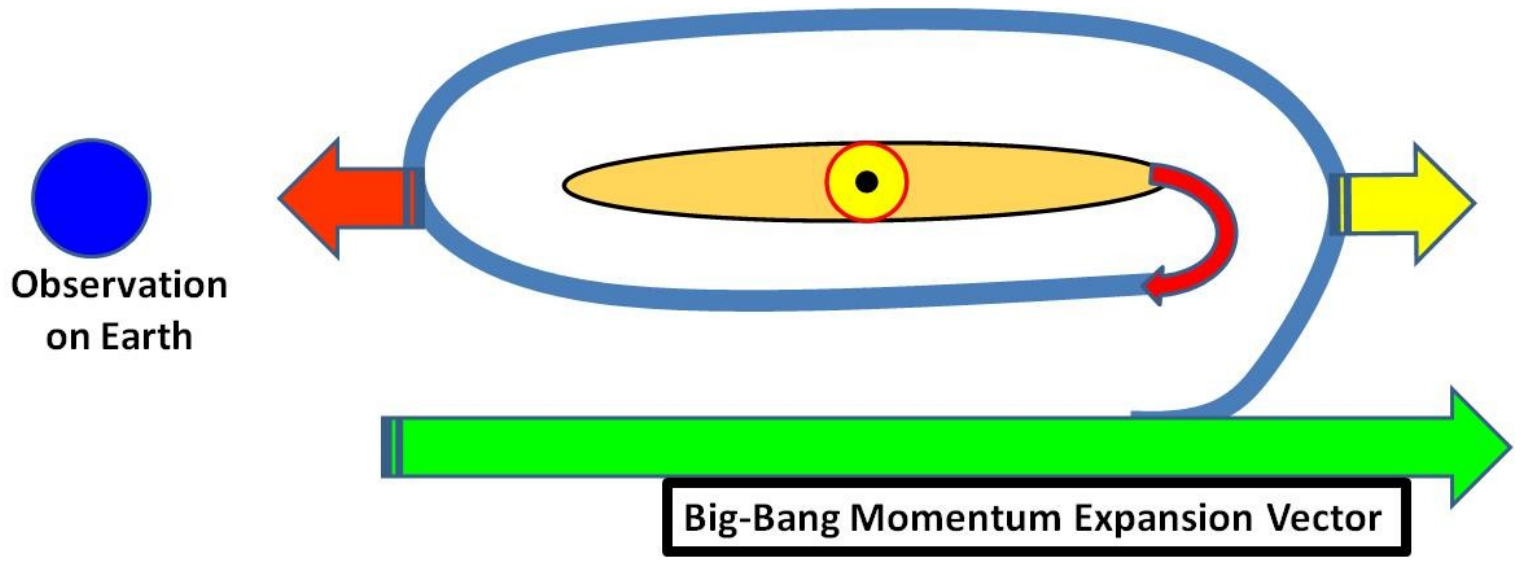


Figure 4 shows the top (or bottom) elliptical boundary face of Kinematic Model 2; while the entire galaxy is proposed to be expanding due to decay of the NOEMKV force-field (after peaking in the formation period billions of years ago), the expansion of the spiral arms was noted as being the greatest where the NOEMKV force-field was the weakest (the major axis of the ellipse). In this diagram the yellow arrow depicts the galactic momentum expansion of that portion of the spiral arm aligned with the Big-Bang momentum expansion vector so they would be additive and increase the rate of Universal Expansion as measured by an observer on Earth. The red arrow depicts the galactic momentum expansion of that portion of the spiral arm aligned against the Big-Bang momentum expansion vector; and while the Big-Bang vector is greater such that all of the galaxy is net moving away from Earth, the measured Universal Expansion rate would be decreased by the spiral arm momentum expansion at this part of the spiral arm.

This could explain variations in measurements of the expansion of the Universe; although it is an expansion in all directions, it is not a constant rate of expansion because (1) the organization and kinematics of galactic formation slowed the process at variable rates depending on the NOEMKV force-field physics/net galactic momentum vector relative to the "Big-Bang momentum vector" for each galaxy, and (2) the orientation of the subsequent localized galactic expansion momentum affects the observed rate of expansion for things like the various positions of spiral arms as demonstrated in Figure 4. The closer a galaxy is to Earth, the more the latter effect would be realized, which is in accordance with the Local Cluster galaxies not fulfilling the Hubble-constant expansion ${ }^{14}$ (though NOEMKV disagrees with the idea that it is "mass-based gravity influenced" in favor of the reasons stated above); this difference would be a smaller percentage of the distance (and therefore a smaller percentage of the expansion rate) the further a celestial object/system is away from the Earth.

The tornado-vortex analogy is again useful here. If the Big-Bang momentum vector is modeled as straight-line wind with velocity vector moving radially-transverse away in every direction away from the Big-Bang, then the formation of any vortex wind would alter the net direction and magnitude of the velocity vector due to the rotational component of velocity. This change would slow the radially transverse Big-Bang momentum vector at locations where the vectors are net contrary, and increase it where the vectors are net complementary, though with everything still moving radially transverse because the Big-Bang momentum vector is larger. It is interesting to contemplate what the cosmic-analogy would be that could cause "straight-line radial Big-Bang winds (cosmic matter)" to begin to rotate (as straight-line winds on Earth can be deflected and organized to rotation and form tornadoes). Was it a shock-wave effect? Or some type of force/wall of pressure that the cosmic matter ran into? This could be a great arena of study for experts in the field. After cosmic rotation was achieved, the fire-tornado model of heat-driven and electrostatics of collisions to increase the organization and intensity (as long as fuel is available to do so) seems to be a logical choice.

The decay of galactic NOEMKV force-fields (and any momentum vector components that had been acting net contrary to the "Big Bang momentum vector") as galaxies have aged is reenabling expansion of the Universe at an accelerating rate (e.g. closer to that imparted by the Big Bang). This is NOEMKV force-field theory's alternative to the expansion of the Universe being caused by "dark energy" that while unknown is generally classified as a repulsive force acting contrary to "gravity." NOEMKV force-field theory offers viable alternative to replace the 
concept of celestial objects attracting each other over vast distances of space due simply to their mass (and classical gravity theory of masses attracting each other with force proportional to the product of their masses- that Einstein displaced at the cosmic scale and which NOEMKV can enhance even further).

Instead of attraction/collapse based on magnitude of mass-based gravity, NOEMKV offers the attraction and organization of matter from heat and electrostatic-driven vortex forces during formation (of galaxies and stars/solar systems), eyes of vortex (that became the center of space around which stars/solar systems orbit e.g. the "barycenter region of solar systems", and became the center of space around which galaxies orbit e.g. the "black hole at the center of galaxies"), colossal electromagnetic field within the vortex spawning tremendous magnetic pressure acting primarily in the $+Z$ and $-Z$ axis to keep matter confined to $\sim 2 \mathrm{D}$ planar kinematics during the formation process and thereafter, imparting the kinematics of the net $\theta$-plane NOEMKV forcefield vector to matter at the time it systematically escaped from the weakest point of the galactic core vortex boundary surfaces (explaining the observed kinematics of stars throughout a galaxy and leading to spiral arms in many galaxies where these forces created semi-bound orbits), and decay of NOEMKV force-fields at the galactic and star-formation level as matter escapes + readily available fuel (hydrogen and dust for heat and electrostatic friction) is consumed and matter cools/coalesces (decreasing the electric field, magnetic field, magnetic pressure, and kinetic energy of formation-vortices). The organization and strengthening of galactic NOEMKV force-fields during formation would explain a slowing of Universal expansion in the past, and the weakening of galactic NOEMKV force-fields as galaxies age explains an accelerating rate of Universal expansion.

To complete the physics background and support of the NOEMKV theory applied to spiral galaxy formation and evolution, a data graph from the Harvard-Smithsonian Center for Astrophysics ${ }^{15}$ may substantiate the existence of the galactic vortex and effects of the proposed galactic NOEMKV force-field on the galactic core and spiral arms of the Milky Way. These are observations and interpretations that have not been discussed with the Center as yet:

- The velocity of CO gas (as shown by spectral line emissions) versus longitude from the galactic core reveals what appears to be a 2-D slice of a cylindrical shape with stretched top and bottom faces (that appears as an hour-glass shape in a 2-D side-view slice) very much in accordance with Kinematic Model 2 of this paper. The stretched-cylindrical slice (hour-glass shape) is tilted about the central velocity axis; if a Z-axis is placed at the center of this cylinder and extends $+\hat{Z}$ and $-\hat{Z}$ along the height axis of the cylinder with $\theta$-plane axis azimuthal about the $Z$ axis, the region of highest velocity (white color in this graph) corresponds to the region around the Z-central point and the region of second highest velocity (red color in this graph) extends from the top to bottom of the vortex core region, as predicted with the fire-tornado vortex model (centripetal acceleration increasing as distance to the center of the vortex decreases)

- At the boundary conditions of the top and bottom faces of the vortex cylinder with cold space, a geometric stretch of galactic matter is observed. In accordance with the proposed Kinematic Model 2, as the NOEMKV force-field weakens at these boundaries the cylinder's faces become stretched; the $\theta$-plane axis that is most stretched becomes the major axis of an ellipse and the orthogonal $\theta$-plane axis that is least stretched becomes 
the minor axis (note that this would require a "top and bottom view" of the galaxy in addition to the "tilted side view" that this galactic plot appears to represent if our interpretation is correct; a "top or bottom view" is present in NASA observations ${ }^{16}$ that show a stretched elliptical region referred to as the Galactic Bar around the galactic core from which the spiral arms originate)

- The stretched top and bottom faces of the vortex would be the locations furthest from the maximum intensity Z-central point of the vortex, and this is exactly where the spiral arms originate (matching the weakest-point of the NOEMKV force-field at which accretion matter would be systematically escape according to the proposed models)

- The galactic matter is observed to remain below the top and bottom faces for a large cosmic distance from the cylindrical region. This is in accordance with theory that all of the $\sim 2 \mathrm{D}$ planar celestial mass in the Milky Way was part of / greatly influenced by the galactic core during the galactic formation period such that galactic core's NOEMKV force-field imparted the $+\hat{Z}$ and $-\hat{Z}$ constrained kinematics to all escaping matter at the time it escaped and formed the spiral arms of the Milky Way.

Interestingly, one spiral arm originates from the stretched top surface and the other spiral arm originates from the stretched bottom surface though at 180 degrees around the perimeter relative to the top arm. It is common to see the spiral arms separated by 180 degrees about a galactic core region, though this plot suggests that there is one point of minimum force-field strength that originates the systematic escape of galactic matter into a spiral arm at the top boundary surface and another point of minimum force-field strength that originates the systematic escape of galactic matter at the bottom boundary face of the galactic vortex that is 180 degrees about the galactic core perimeter. The spiral arms themselves are as far-removed as they can be; this is expected to be a very significant clue though at present time no hypothesis is offered and suggestions are welcome.

It is also a puzzle as to what would cause the boundary faces of the galactic core vortex to stretch to elliptical form. It is hoped that other astrophysicists and researchers can add insight to this and enhance the theories and models in any other ways as well.

\section{Authors' Notes:}

This paper is the third in a sequence that started as the foundation for my son Henry's senior year (high school) honors scientific investigation project. We both have a fascination with astrophysics, especially after attending a show at the California Academy of Sciences Morrison Planetarium in San Francisco and the thought-provoking illustrations and work of so many great scientists in the book "Science- The Definitive Visual Guide" referenced throughout these works. Henry came up with the fire-tornado vortex concept after reading about and watching news on the powerful wildfires in the Western US and tying that to the illustrations in the Science book on star-formation. It is a logical explanation as to "how would a cosmic vortex gain the energy needed to organize and intensify?" The heat from the friction of rotating gas and dust can feed a heat-driven vortex, and the electrostatics can create both organized-attraction of matter and an electric field (and spawn a corresponding magnetic field and magnetic pressure) within. Electromagnetism came to the forefront quickly in our modeling based on the perpendicular 
plasma jets in star formation illustrations in the Science book, as mentioned in our first paper). That's how we came up with the basic concept of NOEMKV force-field theory together; additional study and thought led to the details provided in the three papers.

Henry wrote the computational algorithms for the first two papers and is working on coding exciting 4-D simulations and enhanced algorithms to help demonstrate the concepts visually. Utilizing computer simulations will also help illustrate the 4-D space-time behavior of the concepts, something that can be difficult in the 2-D plane of a computer screen (or pieces of paper the content is printed on with long-winded explanations!). Regardless of whether any of the theories are correct, this project is meant to be a great experience for him to expand his coding skills to object-oriented programming utilizing mathematical equations / computational modeling of real-world problems to enhance his already formidable video-game programming / digital art creation skills that he has been rigorously developing and honing since age 11 .

The NOEMKV concept seems to make logical sense based on the factors discussed and the extent of our knowledge, and can explain as-yet unexplained phenomenon by focusing on the formation period as we have done in all three papers. The concept of kinetic-vortex combined with electromagnetism within is a viable alternative to gravitational-collapse theory though it is acknowledged such alternative is not likely to be welcomed in the astrophysics community. We hope our theories are considered in the spirit of Einstein who left the door open for alternatives in pursuit of maximum scientific truth, was not confronted with gravity-field anomalies that are at present explained with unknown media (dark matter/energy), and his view that "our experience hitherto justifies us in believing that nature is the realization of the simplest conceivable mathematical ideas." 17 NOEMKV force-field theory utilizes well-known forces observed on Earth though in a novel way applied to celestial kinematics, so in our opinion it is more tangible than gravity (since Einstein himself stopped short of explaining "how exactly gravity works" 18 there appears to be potential for enhanced understanding to any theory conventionally attributed to gravity). The goal is not to rain on anyone's parade of past work (or Noble prizes) as diligent and painstaking celestial measurements are still valid, though perhaps just for different reasons/explanations in some cases. Considering that humanity can create vortex and electromagnetism forces for potential beneficial uses perhaps far more than "gravitational forces", applying the vast work of past and present astrophysicists in the context of NOEMKV can probably lead to more practical benefits and greater legacy for such efforts. As discussed in our second paper, the most ideal output of the NOEMKV force-field theory that we are currently aware of is to assist humanity harvest the power of nuclear fusion as a clean and unlimited energy source and/or develop improved motors/rocket-engines as an alternative to combustion processes; any enhanced understanding of celestial formation and astrophysics may help and we hope that our theories may contribute in some way.

All original content herein (C) James Bushong and Henry Bushong

\section{References:}

${ }^{1}$ https://sites.astro.caltech.edu/ george/ay20/Chiappini-MilkyWay.pdf Chiappini, C., The Formation and Evolution of the Milky Way, American Scientist, November/December 2001, pp. 506-515. C 2001 by American Scientist 
${ }^{2}$ Bushong, H., Bushong, J. Theories on the Formation and Evolution of $\sim 2 D$ Planar Celestial Kinematics OSF Preprints

${ }^{3}$ Bushong, H., Bushong, J Theories on the Formation and Evolution of Black Holes \& Galaxies OSF Preprints

${ }^{4}$ https://www.britannica.com/place/Milky-Way-Galaxy/The-structure-and-dynamics-of-theMilky-Way-Galaxy

${ }^{5}$ Born, M., Einstein's Theory of Relativity Dover Publications, 1965

${ }^{6}$ Bushong, H., Bushong, J Theories on the Formation and Evolution of Black Holes \& Galaxies OSF Preprints

${ }^{7} \mathrm{https}: / /$ solarsystem.nasa.gov/resources/285/the-milky-way-galaxy/

${ }^{8}$ Bushong, H., Bushong, J. Theories on the Formation and Evolution of Black Holes \& Galaxies (Figure 8), OSF Preprints

${ }^{9}$ Bushong, H., Bushong, J. Theories on the Formation and Evolution of $\sim 2 D$ Planar Celestial Kinematics OSF Preprints

${ }^{10}$ Science- The Definitive Visual Guide, p.398, DK Publishing 2011 (American Edition)

${ }^{11}$ Fleisch, D., Kregenow, J., A Student's Guide to the Mathematics of Astronomy, p.180, Cambridge University Press 2013

${ }_{12}^{12}$ Science- The Definitive Visual Guide, p.399, DK Publishing 2011 (American Edition)

${ }^{13}$ Ibid., p.328

${ }^{14}$ Fleisch, D., Kregenow, J., A Student's Guide to the Mathematics of Astronomy, p.176, Cambridge University Press 2013

${ }^{15}$ Dame, T.M., Hartmann, D., Thaddeus, P., "The Milky Way on Molecular Clouds: A New Complete CO Survey", The Astrophysical Journal, September 2000

${ }^{16} \mathrm{https}$ ://solarsystem.nasa.gov/resources/285/the-milky-way-galaxy/

${ }^{17}$ Einstein, A., Ideas and Opinions, Three Rivers Press 1954

${ }^{18}$ Science- The Definitive Visual Guide, p.109, DK Publishing 2011 (American Edition) 